\title{
Introduzione al diritto brevettuale italiano
}

\author{
Niccolò Ferretti und Alessandro Zito
}

\section{Introduzione}

In Italia non esiste una vera e propria definizione di invenzione. Non ve ne è traccia né nella legge invenzioni del 1939 né all'interno del Codice della Proprietà Industriale (di seguito C.P.I.). Soltanto l'art. 2585 del codice civile elenca una serie di invenzioni brevettabili. La dottrina più illustre, dunque, ha considerato l'invenzione come ,la soluzione originale di un problema tecnico“ e tale formula può, ancora oggi, essere condivisa. ${ }^{1}$

La definizione sopra indicata sottolinea il fatto che l'invenzione non è determinata da un'idea astratta, ma coincide con la declinazione concreta di tale idea astratta che consiste appunto nel superamento di un problema tecnico. ${ }^{2}$

\section{L'oggetto del brevetto}

L'art. 45 C.P.I. enuclea una serie di realtà che non possono essere considerate come invenzioni e tale elenco è da ritenersi tassativo. Le basi di causa di tale „lista nera“ sono da ritrovarsi principalmente nella logica secondo cui una parte delle realtà

\footnotetext{
${ }^{1}$ Vanzetti - Di Cataldo Manuale di Diritto Industriale, Ed. 2012 Giuffrè; Sena I, 95; ID., R. d. ind. 00 I, 69; Floridia, in Aa Vv., Diritto industriale, pag. 185.

${ }^{2}$ Marchetti - Ubertazzi, Commentario breve alle leggi su Proprietà Intellettuale e Concorrenza, Sesta Edizione, CEDAM 2016, pag. 368.
}

N. Ferretti $(\bowtie) \cdot A$. Zito

Nunziante Magrone Studio Legale Associato, Milano, Italien

E-Mail: n.ferretti@nmlex.it; a.zito@nmlex.it 
appartenenti a tale elenco non sono da considerarsi vere e proprie „,invenzioni“ mentre un' altra parte sono escluse dalla brevettabilità per ragioni di politica legislativa. ${ }^{3}$

L'esclusione dalla brevettazione dei „programmi per elaboratori“ ha una logica sui generis. A metà degli anni ' 60 il software era ancora una materia poco conosciuta, gli uffici brevetti nazionali non sarebbero stati in grado di gestire questa tipologia di invenzioni ed i produttori di Hardware, infine, erano convinti che i software avrebbero compromesso le loro vendite. Ad oggi il software è protetto dal diritto d'autore (legge 22 aprile 1941 n. 633), ma anche il diritto brevettuale ha ammesso la brevettazione di tale trovato a condizione che:

- l'invenzione permetta di comandare il funzionamento di unità di elaborazione $\mathrm{o}$ unità periferiche di sistema,

- l'invenzione sia caratterizzata da un'applicazione dell'informatica alla soluzione di problemi tecnici esterni al computer.

\section{Tipologia di invenzioni}

Possiamo, inoltre, suddividere le invenzioni in 2 macro aree: le invenzioni di prodotto, che hanno ad oggetto un prodotto materiale, e le invenzioni di procedimento, le quali consistono, invece, in una particolare tecnologia di produzione di un bene o di realizzazione di un servizio. Un'altra tipologia di invenzioni sono le c.d. invenzioni derivate ossia quelle di traslazione, combinazione e perfezionamento.

„Invenzione dipendente“, infine, è definita quell'invenzione che, per il suo sviluppo, necessita dell'uso di un prodotto o di un procedimento tutelato da un brevetto anteriore: si tratta dunque di invenzioni di perfezionamento e di combinazione. La „dipendenza“ cosi come la „derivazione“ assumono particolare ed estrema rilevanza soprattutto nell'ambito della biotecnologia. ${ }^{4}$

\section{Requisiti di brevettabilità}

La disciplina brevettuale, per quanto vasta, è piuttosto armonizzata, se non altro per quanto concerne i principi cardine, in particolare, per quanto concerne i requisiti necessari per la brevettazione. Trattasi della novità, dell'altezza inventiva e dell'industrialità.

Nella fattispecie, per quanto concerne la novità, essa è un requisito dell'invenzione brevettabile e consiste nella differenza formale tra l'invenzione di cui si domanda il riconoscimento del titolo e lo stato della tecnica rilevante. Viene talvolta

\footnotetext{
${ }^{3}$ Vanzetti - Di Cataldo Manuale di Diritto Industriale, Ed. 2012 Giuffrè.

${ }^{4}$ Più approfonditamente Alberto Musso, Il rapporto di dipendenza fra invenzioni biotecnologiche (o con prodotti e materiali da esse „,derivati),“"Rivista di Diritto Industriale fasc. 6, 2014, pag. 289.
} 
definita come novità estrinseca per evidenziarne la differenza e la complementarietà con il requisito della non ovvietà, indicato a sua volta come novità intrinseca, e consistente nel fatto che l'invenzione non sia sostanzialmente simile a ciò che è compreso nello stato della tecnica. ${ }^{5}$

Lo stato della tecnica a cui bisogna far riferimento ai fini del giudizio di novità è correlato ad una nozione di novità assoluta ed universale priva di confini spaziali e territoriali. Rientra nell' ambito dello stato della tecnica:

i) la common general knowledge,

ii) la enhanced knowledge,

iii) la hidden knowledge, e iv) la prior application. ${ }^{6}$

Elementi distruttivi della novità sono la predivulgazione e le anteriorità invalidanti. Per quanto concerne la predivulgazione, prima del deposito della domanda di brevetto, l'invenzione si intende divulgata al pubblico dallo stesso inventore o da un terzo. La predivulgazione deve essere rivolta ad un numero indeterminato di persone che siano in grado di comprendere l'informazione. ${ }^{7}$

Una divulgazione non inficia la novità della invenzione se si è verificata nei sei mesi che precedono la data di deposito della domanda di brevetto e risulta direttamente o indirettamente un abuso evidente ai danni del richiedente o del suo dante causa. Non è presa, altresì, in considerazione la divulgazione avvenuta in esposizioni ufficiali o ufficialmente riconosciute ai sensi della Convenzione di Parigi del 22 novembre 1928 e successive modifiche, concernente le esposizioni internazionali.

L'anteriorità invalidante, invece, è un documento anteriore che riproduce integralmente i medesimi elementi dell'invenzione. L'anteriorità che anticipa completamente gli insegnamenti della invenzione brevettanda e consiste in un unico documento determinerà l'assenza di novità. ${ }^{8}$

La capacità distruttiva della novità in virtù di un documento anteriore si profila anche nel caso in cui tale capacità possa essere determinata a seguito della combinazione fra gli elementi presenti nell'anteriorità ed elementi rinvenibili nella conoscenza generale comune (common general knowledge).

Trattiamo ora del secondo requisito di brevettabilità, l'altezza inventiva. Un'invenzione è considerata come implicante un'attività inventiva, se, per una persona esperta del ramo, essa non risulta in modo evidente dallo stato della tecnica. Ad oggi, il requisito dell'attività inventiva è ormai positivizzato e parametrato alla c.d. „,non evidenza per la persona esperta del ramo“.

\footnotetext{
${ }^{5}$ Definizione tratta da Marchetti - Ubertazzi, Commentario breve alle leggi su Proprietà Intellettuale e Concorrenza, 6 edizione, Ed. Cedam 2016, pag. 368.

${ }^{6}$ Suddivisione tratta da Marchetti - Ubertazzi, Commentario breve alle leggi su Proprietà Intellettuale e Concorrenza, 6 edizione, Ed. Cedam 2016, pag. 369.

${ }^{7}$ Piola - Caselli, Privativa, Nuovo D., n. 22; Ascarelli, Teoria della Concorrenza e dei Beni Immateriali, pag. 550; Greco - Vercellone, Le invenzioni ed i modelli industriali, in Trattato dir. civ., pag. 114; Trib. di Genova 29.04.1971; Trib. di Torino 22.01.1979; App. Milano 11.07.1975 in GADI 75, pag. 558; Trib. di Milano 18.10.1973 in GADI 1973, pag. 1201.

${ }^{8}$ Corte di Appello di Milano, 28.02.2003 in GADI 2003, pag. 799.
} 
Con chi si identifica la persona esperta del ramo? Egli deve essere un operatore del settore, il quale tuttavia dovrà avere le capacità che mediamente gli operatori possiedono nel settore di riferimento (l'esperto del ramo non dovrà quindi né eccellere, né avere scarse doti intellettive e culturali). ${ }^{9}$

La più accorta dottrina evidenzia che, a seguito di una tendenza sempre maggiore da parte dell'Ufficio Italiano Marchi e Brevetti a concedere brevetti che spesso, a seguito di una verifica ex post, si rivelano essere nulli, si dovrebbe rielaborare il concetto di ,persona esperta del ramo“.

In particolare, ci si chiede come mai resti ancora oscura la ragione per cui l'esperto del ramo non sia da individuarsi nella figura di un ricercatore, di un supertecnico, o di un team di ricercatori, nel caso delle c.d. invenzione di equipe. ${ }^{10}$

L'ultimo requisito necessario per brevettare un'invenzione è quello dell'industrialità. Un'invenzione, infatti, è considerata atta ad avere un'applicazione industriale, se il suo oggetto può essere fabbricato o utilizzato in qualsiasi genere di industria, compresa quella agricola. Tale requisito attiene all'idoneità del risultato inventivo ad essere concretamente applicato, mentre nulla ha a che vedere con la convenienza economica della sua attuazione.

L'utilità dell'invenzione non va intesa in senso patrimoniale, ma in relazione al progresso della tecnica ed al soddisfacimento dei bisogni umani: il suo valore economico è irrilevante ai fini della concessione della privativa. Sono perciò brevettabili anche le invenzioni la cui applicazione industriale non sia conveniente, sotto il profilo del costo, comparativamente al presumibile vantaggio. ${ }^{11}$

Spiegato in termini teorici cosa è l'industrialità, in termini pratici, il requisito de $q u o$, altro non è se non la c.d. utilizzabilità industriale. L'invenzione, dunque, deve proporsi uno scopo teoricamente raggiungibile. In giurisprudenza, tale requisito è stato indagato soltanto rarissime volte.

\section{Diritti nascenti dal brevetto}

Una volta ottenuta la concessione del brevetto, numerosi sono i diritti nascenti da tale diritto di privativa. E' fondamentale sottolineare che secondo il diritto italiano alcuni diritti di brevetto scaturiscono già dal brevetto allo stato di domanda. Nella fattispecie, gli effetti del brevetto decorrono dalla data in cui la domanda con la descrizione, le rivendicazioni e gli eventuali disegni è resa accessibile al pubblico. La pubblicazione avviene decorsi 18 mesi dalla data di deposito. In caso di volontà da parte del titolare di rendere la domanda accessibile al pubblico prima dei 18 mesi, egli può inoltrare un'istanza di pubblicazione anticipata.

\footnotetext{
${ }^{9}$ Definizione tratta da Adriano Vanzetti, Codice della proprietà Industriale, Ed. Giuffrè 2013, pag. 668.

${ }^{10}$ Sara Grisanti, L'esperto del ramo e l'attività inventiva, in Il Diritto Industriale 1/2019, Ed Ipsoa, pag. 20.

${ }^{11}$ Corte di Appello di Milano, 17 giugno 1994 in GADI 1980, pag. 52.
} 
Qualora il titolare della domanda desideri che gli effetti della domanda di brevetto vengano prodotti nei confronti di uno specifico soggetto, il titolare della domanda potrà procedere alla notifica nei confronti del soggetto in questione. Gli effetti decorrono, in tal caso, dalla data della notifica.

Come abbiamo evidenziato all'inizio della trattazione, non vi è una definizione univoca di ,invenzione“, mentre il brevetto può essere definito come l'istituto giuridico attraverso il quale l'ordinamento assicura all'inventore il diritto di utilizzazione esclusiva dell'invenzione per un certo periodo di tempo. ${ }^{12}$

L'art. 78 E.P.C. e l'art. 51 comma 1 C.P.I. statuiscono di quali parti si compone il brevetto. Prima fra tutte, il riassunto, il quale ha il fine di determinare quale sia l'oggetto dell'invenzione. La descrizione è un'altra parte imprescindibile del brevetto (art. 51 del C.P.I. e art. 42 del Regolamento di esecuzione E.P.C.). Nella fattispecie, la descrizione deve:

a) precisare il ramo della tecnica al quale l'invenzione si riferisce;

b) indicare lo stato anteriore della tecnica nella misura in cui, a conoscenza del richiedente, esso possa essere considerato utile per l'intelligenza dell'invenzione, per la redazione del rapporto di ricerca europea e per l'esame della domanda di brevetto europeo; preferibilmente, si citeranno le fonti da cui risulta lo stato anteriore della tecnica;

c) esporre l'invenzione, quale è caratterizzata nelle rivendicazioni, in termini tali che si possa comprendere il problema tecnico, anche se esso non è esplicitamente designato come tale, e la sua soluzione; indicare inoltre gli eventuali vantaggi procurati dall'invenzione rispetto allo stato anteriore della tecnica;

d) descrivere brevemente le figure contenute nei disegni, se ve ne sono;

e) indicare dettagliatamente almeno un modo di attuazione dell'invenzione per la quale la protezione è richiesta; tale indicazione deve riportare esempi, se del caso, e riferimenti ai disegni, se ve ne sono;

f) indicare esplicitamente, se ciò non risulta in modo evidente dalla descrizione o dalla natura dell'invenzione, in qual modo quest'ultima è atta ad avere un'applicazione industriale.

Parte cruciale del brevetto sono le sue rivendicazioni (art. 52 C.P.I. ed art. 69 E.P.C.). Nelle rivendicazioni è indicato, specificamente, ciò che si intenda debba formare oggetto di brevetto. I limiti della protezione sono determinati dalle rivendicazioni, tuttavia la descrizione ed i disegni servono ad interpretare le stesse.

Ai fini dell'individuazione del nucleo tutelabile del brevetto occorre fare riferimento esclusivamente alle rivendicazioni, che si pongono quale vera e propria manifestazione di volontà con la quale il richiedente identifica lo specifico ambito di protezione del brevetto. Le rivendicazioni sono suscettibili di interpretazione alla luce di descrizione e disegni, sicchè la tutela brevettuale va circoscritta a ciò che risulta contemporaneamente rivendicato e descritto. ${ }^{13}$ La rivendicazione, pertanto,

\footnotetext{
${ }^{12}$ Vanzetti - Di Cataldo, Manuale di Diritto Industriale, Ed. Giuffrè, 2012, pag. 357.

${ }^{13}$ Tribunale di Milano, 29.03.2007 in GADI 2009, pag. 305.
} 
costituisce l'identificazione della specifica protezione che si pretende. ${ }^{14}$ Per quanto concerne i disegni, essi, per la normativa italiana, devono soggiacere a determinate formalità (Art. 22 Reg. att. C.P.I.).

Torniamo dunque ai diritti scaturenti dal brevetto. Primo fra tutti, il diritto di essere riconosciuto autore. In particolare il diritto di essere riconosciuto autore dell'invenzione può essere fatto valere dall'inventore e, dopo la sua morte, dal coniuge e dai discendenti fino al secondo grado; in loro mancanza o dopo la loro morte, dai genitori e dagli altri ascendenti ed in mancanza, o dopo la morte di questi, dai parenti fino al quarto grado incluso.

Per quanto riguarda, invece, i diritti patrimoniali nascenti dal brevetto (tranne il diritto ad essere riconosciuto autore), questi sono alienabili e trasmissibili, a differenza del diritto morale. Se l'invenzione è stata realizzata da un gruppo di persone, le facoltà relative sono regolate, salvo convenzioni in contrario, dalle disposizioni del codice civile in tema di comunione.

In caso di diritto appartenente a più soggetti, la presentazione della domanda di brevetto, la prosecuzione del procedimento di brevettazione, la presentazione della domanda di rinnovo, il pagamento dei diritti di mantenimento in vita, la presentazione della traduzione in lingua italiana delle rivendicazioni di una domanda di brevetto europeo concesso o mantenuto in forma modificata o limitata e gli altri procedimenti di fronte all'Ufficio Italiano Marchi e Brevetti possono essere effettuati da ciascuno di tali soggetti nell'interesse di tutti. I contitolari sono, però, obbligati in solido al pagamento delle tasse di brevetto.

\section{Le invenzioni dei dipendenti}

Un breve accenno merita anche la disciplina delle invenzioni dei lavoratori dipendenti. Nella fattispecie distinguiamo 3 tipologie:

- le invenzioni di servizio;

- le invenzioni di azienda;

- le invenzioni occasionali.

Nella prima tipologia si profila il caso in cui l'invenzione è posta in essere dal lavoratore dipendente che svolge attività di lavoro di ricerca volta all'invenzione. Lo svolgimento della predetta attività lavorativa è parte delle attività oggetto del contratto di lavoro. Infine, l'attività lavorativa in questione deve essere oggetto di specifica retribuzione.

Se i summenzionati criteri sono rispettati, la titolarità dell'invenzione di servizio, con gli annessi diritti patrimoniali, sarà di titolarità del datore di lavoro, salvo il diritto dell'inventore di essere riconosciuto autore.

Per quanto concerne le invenzioni di azienda, la disciplina è lievemente differente. Nel caso di specie, l'attività inventiva non è oggetto del contratto che lega il

${ }^{14}$ Corte di Cassazione 01.09.1997 n. 8324 in banca dati Pluris on Line. 
dipendente al datore di lavoro e l'invenzione viene realizzata in esecuzione dell'adempimento di un contratto di lavoro. La titolarità dell' invenzione, con i relativi diritti patrimoniali, spetterà al datore di lavoro, salvo il diritto del lavoratore di esserne riconosciuto autore e ricevere un equo premio.

Nella fattispecie, nel momento in cui una invenzione viene qualificata come ,invenzione di azienda" comporta il diritto per il dipendente, inventore del trovato, di ricevere da parte del datore di lavoro un equo premio ,tenendo conto dell'importanza dell'invenzione, delle mansioni del svolte e della retribuzione percepita dall'inventore, nonché del contributo che questi ha ricevuto dall'organizzazione del datore di lavoro".

Sul punto la maggior parte della giurisprudenza ${ }^{15}$ ritiene che l'elemento distintivo fra le due ipotesi (invenzione di servizio e le invenzioni di azienda) risiede precipuamente nella presenza o meno di una esplicita previsione contrattuale di una speciale retribuzione costituente corrispettivo dell' attività inventiva. La dottrina ha osservato, però, che le previsioni contrattuali relative all'equo premio darebbero vita, nel lungo periodo, a comportamenti calcolatori da parte dei datori di lavoro che potrebbero ,insinuare“ nel contratto clausole relative ad una specifica retribuzione per attività inventiva nei confronti di tutti i dipendenti, anche per coloro che non hanno mai svolto attività inventiva.

Una questione dibattuta è determinata dal dies a quo da cui nasce il diritto all' equo premio (diritto su cui opera il termine di prescrizione ordinario di 10 anni ex art. 2946 c.c.). La giurisprudenza e lo stesso dettato normativo si è attestata sull'orientamento secondo cui l'equo premio spetta al dipendente qualora il datore di lavoro o i suoi aventi causa ottengano il brevetto o utilizzino l'invenzione in regime di segretezza industriale. ${ }^{16}$

La terza ed ultima categoria, le c.d. invenzioni occasionali, di contro, è realizzata dal dipendente al di fuori del rapporto di lavoro, pur rientrando nel campo di attività del datore di lavoro. Il diritto morale e patrimoniale scaturente dall'invenzione spetta all'inventore, anche se il datore di lavoro ha il diritto di opzione per l'uso esclusivo e non esclusivo dell'invenzione o per l'acquisto del brevetto.

Tale opzione sussiste anche per la facoltà di chiedere o acquisire, per la medesima invenzione, brevetti all'estero verso corresponsione del canone o del prezzo da fissarsi con deduzione di una somma corrispondente agli aiuti che l'inventore abbia comunque ricevuto dal datore di lavoro per pervenire all'inventore. Il diritto di opzione va esercitato entro 3 mesi dalla data di ricevimento della comunicazione dell'avvenuto deposito della domanda di brevetto.

\footnotetext{
${ }^{15}$ Una su tutte: Tribunale di Milano, 28 ottobre 2015 n. 12048 in banca dati Pluris On Line.

${ }^{16}$ Più ampiamente, sul punto: Carmine di Benedetto, Il Diritto Industriale 4/2016 Ed. Ipsoa, pag. 327 e ss.
} 


\section{Le invenzioni dei ricercatori di università ed enti pubblici di ricerca}

È possibile che l'invenzione venga realizzata da ricercatori di università ed enti pubblici di ricerca. In questo caso, la disciplina concernente le università si applica a quelle pubbliche e private. È possibile suddividere la categoria delle invenzioni di ricercatori di università ed enti pubblici di ricerca nelle sottocategorie della c.d. ricerca istituzionale e della ricerca c.d. finanziata. Per quanto concerne la prima sottocategoria, i diritti derivanti dall'invenzione istituzionale spettano al ricercatore, che dovrà rendere edotto l'ente di appartenenza dell'avvenuto deposito della domanda di brevetto.

Le università e le pubbliche amministrazioni, nell'ambito della propria autonomia, stabiliscono l'importo massimo del canone, relativo a licenze ai terzi per l'uso dell'invenzione, spettante alla stessa università o alla pubblica amministrazione ovvero ai privati enti finanziatori della ricerca. Nel caso in cui nulla sia stabilito nello Statuto, l'Università, l'ente o la pubblica amministrazione hanno diritto a non meno del $30 \%$ dei proventi o canoni.

Trascorsi 5 anni dalla concessione del brevetto, qualora l'inventore o i suoi aventi causa non ne abbiano iniziato lo sfruttamento industriale (salvo casi indipendenti dalla sua volontà), la pubblica amministrazione di cui l'inventore era dipendente al momento dell'invenzione acquisisce direttamente un diritto gratuito, non esclusivo, di sfruttare l'invenzione ed i diritti patrimoniali ad essa connessi o di farli sfruttare da terzi.

Tale statuizione si basa sul modello c.d. di titolarità individuale, tipico di paesi come Italia e Svezia. Tale modello è stato introdotto in Italia dall'art. 7 della legge n. 383/2001 che inserì nell'allora vigente Legge Invenzioni l'art. 24 bis. Nel Codice della Proprietà Industriale è stata aggiunta una precisazione rispetto alla disciplina precedente, ossia il comma 5 dell' art. 65 (le ricerche finanziate).

Un dibattito dottrinale nacque sulla questione di chi fossero i soggetti che rientravano nella categoria dei ricercatori universitari. Tali possono essere considerati soltanto i professori ordinari ed associati ed i ricercatori, oppure anche i tecnici laureati e di laboratorio i ricercatori para subordinati, come ad esempio assegnisti di ricerca, borsisti post laurea/dottorato? La questione è tutt'oggi fonte di dibattito.

Inoltre, l'adozione del modello della titolarità individuale da parte dell'Italia risiede nella convinzione secondo cui le Università non sarebbero in grado di creare e gestire diritti di proprietà intellettuale. Tale considerazione non può essere in toto condivisa. Infatti, la stessa riflessione può essere svolta per quanto riguarda la figura dei ricercatori, i quali dovrebbero sostenere ingenti costi per la gestione dei diritti di proprietà intellettuale. Si pensi, ad esempio, ai costi di deposito, di pagamento delle tasse annuali, le spese di consulenza di un professionista, ecc. 
Si auspica, dunque, un cambio di rotta al fine di sposare ad esempio il sistema statunitense, dove il ricercatore, l'università ed il laboratorio si dividono i proventi dell'invenzione ed hanno l'impegno di tentare di ,piazzare“ il trovato sul mercato. ${ }^{17}$

La summenzionata disciplina non si applica in caso di attività di ricerca svolta nel quadro di determinati progetti di ricerca finanziati da soggetti privati o pubblici diversi dall'organizzazione di appartenenza.

I diritti di brevetto per invenzione industriale consistono nella facoltà esclusiva di attuare l'invenzione e di trarne profitto nel territorio dello Stato, entro i limiti ed alle condizioni previste dalla legge.

\section{Il principio di esaurimento}

A tal proposito, un altro assioma fondamentale della disciplina brevettuale è caratterizzato dal principio di esaurimento. Il titolare di un brevetto non può opporsi alla circolazione del prodotto oggetto di tutela una volta che esso sia stato immesso in commercio dal titolare del brevetto con il suo consenso. Tale principio si profila ogni qualvolta l'immissione in commercio con il consenso del titolare si verifica in qualsiasi stato dello Spazio Economico Europeo (SEE).

La norma in commento non si applica in caso in cui sussistano motivi legittimi perché il titolare stesso si opponga all'ulteriore commercializzazione dei prodotti, in particolare, quando lo stato di questi è modificato o alterato dopo la loro immissione in commercio. Dopo questa necessaria digressione, torniamo ad esaminare $\mathrm{i}$ diritti esclusivi derivanti dal brevetto.

\section{La contraffazione}

In particolare il brevetto conferisce al titolare il diritto di vietare a terzi, salvo consenso del titolare, di produrre, usare, mettere in commercio, vendere o importare a tali fini il prodotto oggetto di brevettazione.

Se oggetto del brevetto è un procedimento, il brevetto conferisce il diritto di vietare a terzi, salvo consenso del titolare, di applicare il procedimento, nonché di usare, mettere in commercio, vendere o importare a tali fini il prodotto direttamente ottenuto con il procedimento in questione. Trattasi, questi ultimi, di atti di contraffazione.

La dottrina e la giurisprudenza hanno sviluppato vari tipi di contraffazione. Si profila la contraffazione c.d. letterale nel caso in cui il prodotto/procedimento

\footnotetext{
${ }^{17}$ Una visione comparatistica molto dettagliata in Claudia del Re, Il modello di titolarità dei risultati della ricerca universitaria come parametro di efficienza del trasferimento tecnologico accademico: la preferibilità del modello di titolarità istituzionale, Rivista di Diritto Industriale, fasc. 6, 2016, pag. 272.
} 
contraffatto riproduce letteralmente le rivendicazioni brevettuali. Si tratterà di contraffazione definita ,per equivalenti“" nel caso in cui il prodotto/procedimento non riprenda letteralmente la rivendicazione, ma contenga elementi equivalenti a quelli oggetto di rivendicazione. ${ }^{18}$

In particolare il concetto dell'equivalenza è stato positivizzato a livello internazionale nei paesi che hanno ratificato la versione 2000 della Convenzione sul Brevetto Europeo che è entrata in vigore al termine dell'anno 2007. Nel Codice della Proprietà Industriale l'equivalenza viene menzionata nell'art. 52, 3 bis, il quale recita: „per determinare l'ambito della protezione conferita dal brevetto, si tiene nel dovuto conto ogni elemento equivalente ad un elemento indicato nelle rivendicazioni“".

Per individuare l'equivalenza, la giurisprudenza internazionale ha proposto vari criteri, ognuno dei quali risulta però essere carente per una ragione o per un'altra.

Ad oggi il criterio maggiormente recepito, di derivazione statunitense, è quello della c.d. tripla identità. Ossia, un prodotto o un procedimento risulta essere equivalente a quello presente nella rivendicazione se esso:

i) risolve lo stesso problema tecnico;

ii) ottenendo lo stesso risultato;

iii) con mezzi sostanzialmente uguali, ossia sostanzialmente nello stesso modo.

Il concetto di „sostanzialmente“ è quello più opinabile dove si scontrano sistematicamente i tecnici del settore. Pur se non normativamente previsto, il concetto di equivalenza viene contemplato nelle linee guida dell'Ufficio Brevetti Europeo. Un diverso criterio di valutazione dell'equivalenza è determinato dal c.d. giudizio di ovvietà della sostituzione. Se la diversità è il risultato di una modifica ovvia sussiste equivalenza. Spesso la giurisprudenza italiana è solita combinare i criteri sopra indicati. ${ }^{19}$ Un'altra tipologia di contraffazione è quella di tipo „evolutivo“.

$\mathrm{Nel}$ nostro ordinamento tale concetto è positivizzato nell'art. 2587 c.c. il quale afferma che il brevetto per invenzione industriale, la cui attuazione implica quella di invenzioni protette da precedenti brevetti per invenzioni industriali ancora in vigore, non pregiudica i diritti dei titolari di questi ultimi, e non può essere attuato né utilizzato senza il consenso di essi. Lo stesso secondo comma dell'art. 68 C.P.I. statuisce che il brevetto per invenzione industriale, la cui attuazione implichi quella di invenzioni protette da precedenti brevetti per invenzioni industriali ancora in vigore, non può essere attuato, né utilizzato, senza il consenso dei titolari di questi ultimi.

La Legge del 2 novembre 2016, n. 214, che ratifica la Convenzione sul Brevetto ad Effetto Unitario, ha introdotto, nell' art. 66 del Codice della proprietà Industriale, l'istituto del cosiddetto ,contributory infringement“, la c.d. contraffazione indiretta. Fattispecie già analizzata dai nostri giudici della nomofilachia con Sentenza n. 5406

\footnotetext{
${ }^{18}$ Per una visione approfondita e comparatistica: Sandro Hassan, Equivalenza e interpretazione del brevetto in un'ottica sovranazionale, Rivista di diritto industriale, fasc. 4-5, 2013, pag. 209.

${ }^{19}$ Tribunale di Milano 8 agosto 2007 in GADI 2007 n. 5164; Tribunale di Roma 9 febbraio 2006 in GADI 2006 n. 5003; Tribunale di Roma 13 aprile 2006 in GADI 2006 n. 5018; Cassazione Civile 13 gennaio 2004 n. 257 in GADI 2004 n. 4626; Cassazione Civile 5 settembre 1990 n. 9142 in GADI 1990, n. 2477.
} 
del 12.06.2006. Nella fattispecie, il brevetto conferisce al titolare anche il diritto esclusivo di vietare a terzi, salvo proprio consenso, di fornire o di offrire a soggetti diversi dagli aventi diritto all'utilizzazione dell'invenzione brevettata i mezzi relativi ad un elemento indispensabile di tale invenzione e necessari per la sua attuazione nel territorio di uno Stato in cui la medesima sia protetta, qualora il terzo abbia conoscenza dell'idoneità e della destinazione di detti mezzi di attuare l'invenzione o sia in grado di averla con l'ordinaria diligenza.

Tale disciplina non si applica quando i mezzi sono costituiti da prodotti che si trovano correntemente in commercio, a meno che il terzo non induca il soggetto a cui sono forniti a compiere gli atti vietati sopra menzionati. Ci si è chiesti se il contributo alla contraffazione possa considerarsi applicabile soltanto ai brevetti c.d. di procedimento o anche ai brevetti c.d. di prodotto. La risposta sembrerebbe essere affermativa, dato che l'art. 66 del Codice della Proprietà Industriale si riferisce genericamente a „mezzi“, questi ultimi non debbono essere considerati soltanto come dispositivi ma anche come ,fasi“ dell'invenzione.

Inoltre, sempre con riferimento al contributory infringement, il concetto della rilevanza territoriale è di massima importanza. Secondo autorevole dottrina, al fine di fondare pienamente la competenza dell'autorità giurisdizionale dello Stato, il contributo alla contraffazione deve essere svolto in Italia, per una invenzione tutelata in Italia. Dunque, profilandosi l'attività preparatoria di alcuni rilevanti fasi protette dalla rivendicazione (con efficacia in Italia) e la contraffazione diretta e finale avviene in qualunque Paese l'invenzione sia protetta (dunque estero compreso), vi è contributo alla contraffazione ex art. 66 Codice della Proprietà Industriale. ${ }^{20}$

Lo ius exludendi alios conferito dal brevetto non è assoluto ed è temperato da alcune limitazioni. In particolare, non sono da considerarsi illeciti gli atti compiuti in ambito privato con fini non commerciali, gli atti compiuti in via sperimentale, gli atti diretti all'ottenimento dell' AIC (Autorizzazione Immissione in Commercio di un farmaco) ed il preuso segreto.

Con riferimento a quest'ultimo, l'art. 68 comma 3 C.P.I. statuisce che chiunque, nel corso dei dodici mesi anteriori alla data di deposito della domanda di brevetto o alla data di priorità, abbia fatto uso nella propria azienda dell'invenzione, può continuare ad usarla nei limiti del preuso. Tale facoltà è trasferibile soltanto insieme all'azienda in cui l'invenzione viene utilizzata. La prova del preuso e della sua estensione è a carico del preutente.

\section{Cause di estinzione del brevetto}

Vi sono delle cause in cui il brevetto si estingue e con esso i diritti che ne derivano. Nella fattispecie, il brevetto per invenzione dura 20 anni a decorrere dalla data di deposito della domanda e non può essere rinnovato, né può esserne prorogata la

\footnotetext{
${ }^{20}$ Vincenzo Jandoli, Il contributo alla contraffazione, Il Diritto Industriale 1/2017, Ed. Ipsoa, pag. 34.
} 
durata. Secondo la disciplina italiana, il brevetto può anche essere oggetto di rinuncia. In particolare, il titolare può rinunciare al brevetto con atto ricevuto dall'U.I.B.M. da annotare sul registro dei brevetti. Il brevetto può anche essere limitato su istanza del titolare, alla quale devono unirsi la descrizione, le rivendicazioni ed i disegni modificati.

In un giudizio di nullità, il titolare del brevetto ha facoltà di sottoporre al giudice, in ogni stato e grado del procedimento, una riformulazione delle rivendicazioni che rimanga entro i limiti del contenuto della domanda di brevetto quale inizialmente depositata e non estenda la protezione conferita dal brevetto concesso.

Il brevetto può estinguersi anche per decadenza. Essa si profila in caso di mancato pagamento dei diritti che devono essere versati anticipatamente, con cadenza annuale, entro il mese corrispondente a quello in cui è stata depositata la domanda. Il brevetto è soggetto a decadenza anche nel caso in cui si determini una mancata o insufficiente attuazione dell'invenzione. Ciò avviene quando l'invenzione non è stata attuata dal titolare del brevetto e dai titolari di licenze obbligatorie, entro due anni dalla concessione della prima licenza. Tale norma vale anche nel caso in cui né il titolare del brevetto, né i titolari di licenze obbligatorie hanno attuato l'invenzione in modo proporzionato rispetto alle esigenze del paese.

\section{Cause di nullità del brevetto}

Il brevetto può anche essere dichiarato nullo in casi tassativi. In particolare, ciò accade quando l'invenzione non rientra nella materia brevettabile, è carente dei requisiti per la concessione oppure è illecita. Anche l'insufficiente descrizione del brevetto può causare la nullità dello stesso. Ed invero, recente giurisprudenza ${ }^{21}$ ha statuito che nel valutare una domanda di novità la Corte non può esimersi dal verificare contemporaneamente la sussistenza del requisito relativo alla sufficiente descrizione di cui all'art. 51 Codice della Proprietà Industriale. Secondo la Cassazione, il requisito della novità intrinseca ed estrinseca deve obbligatoriamente essere integrato con la chiara ed esauriente indicazione, all'interno del testo brevettuale, del problema tecnico da risolvere rispetto al quale il trovato ne è soluzione. ${ }^{22}$ La medesima nullità colpisce il brevetto che rivendica materia non descritta nella domanda o che è stato concesso ad un non avente diritto.

Si consideri, in ogni caso, che il brevetto nullo può produrre gli effetti di un diverso brevetto del quale contenga i requisiti di validità e che sarebbe voluto dal richiedente, qualora questi ne avesse conosciuto la nullità. La domanda di conversione può essere proposta in ogni stato e grado del giudizio. La sentenza che accerta i requisiti di validità del diverso brevetto dispone la conversione del brevetto nullo. Il titolare del brevetto convertito, entro 6 mesi dal passaggio in giudicato della sentenza di conversione, presenta domanda di correzione del brevetto. Gli effetti della nullità hanno efficacia erga omens et ex tunc.

\footnotetext{
${ }^{21}$ Cassazione Civile, Sez. I 22 novembre 2010, n. 23592 in Banca Dati Pluris on Line.

${ }^{22}$ Cristiano Bacchini, Il Diritto Industriale 5/2011 Ed. Ipsoa, pag. 444.
} 
Sono fatti salvi, ai sensi del combinato disposto degli artt. 77 e 123 C.P.I.:

a) gli atti di esecuzione di sentenze di contraffazione passate in giudicato già compiuti;

b) i contratti aventi ad oggetto l'invenzione conclusi anteriormente al passaggio in giudicato della sentenza che ha dichiarato la nullità nella misura in cui siano già stati eseguiti. In questo caso, tuttavia, il giudice, tenuto conto delle circostanze, può accordare un equo rimborso di importi già versati in esecuzione del contratto;

c) i pagamenti già effettuati ai sensi degli articoli 64 C.P.I. (invenzione dei dipendenti) e 65 C.P.I. (invenzioni dei ricercatori delle università e degli enti pubblici), a titolo di equo premio, canone o prezzo.

Ci si domanda in dottrina se l'azione di nullità di un brevetto sia da inquadrarsi come azione di mero accertamento o sia un'azione costitutiva. L'orientamento prevalente della giurisprudenza è quello secondo cui le domande di nullità brevettuale sarebbero costitutive e assimilabili alle impugnative negoziali. I motivi di nullità devono essere azionati mediante atto di citazione oppure con domanda riconvenzionale. Da ciò deriva che la deduzione di nuove ipotesi di nullità del brevetto rispetto a quelle contemplate negli atti introduttivi del giudizio debbano intendersi come una mutatio libelli.

La conseguenza di tale assunto è determinata dal fatto che se l'attore risulta soccombente in primo grado, potrà instaurare un altro giudizio volto ad ottenere la nullità della stessa privativa sulla base di motivi di nullità diversi da quelli azionati nel processo precedente. ${ }^{23}$

${ }^{23}$ Per una più ampia disamina: Michele Vanzetti, Mutatio libelli nelle domande di nullità brevettuale: le ragioni di una possibile lettura alternativa, Rivista di Diritto Industriale, fasc. 2, 1 aprile 2018, pag. 77.

Open Access Dieses Kapitel wird unter der Creative Commons Namensnennung 4.0 International Lizenz (http://creativecommons.org/licenses/by/4.0/deed.de) veröffentlicht, welche die Nutzung, Vervielfältigung, Bearbeitung, Verbreitung und Wiedergabe in jeglichem Medium und Format erlaubt, sofern Sie den/die ursprünglichen Autor(en) und die Quelle ordnungsgemäß nennen, einen Link zur Creative Commons Lizenz beifügen und angeben, ob Änderungen vorgenommen wurden.

Die in diesem Kapitel enthaltenen Bilder und sonstiges Drittmaterial unterliegen ebenfalls der genannten Creative Commons Lizenz, sofern sich aus der Abbildungslegende nichts anderes ergibt. Sofern das betreffende Material nicht unter der genannten Creative Commons Lizenz steht und die betreffende Handlung nicht nach gesetzlichen Vorschriften erlaubt ist, ist für die oben aufgeführten Weiterverwendungen des Materials die Einwilligung des jeweiligen Rechteinhabers einzuholen.

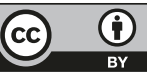

